\title{
A novel fetal sequence patogenesis resulting in oral meningoencephalocele
}

\author{
Gabor $\mathrm{M}^{1}$, Papcun $\mathrm{P}^{1}$, Krizko Jr $\mathrm{M}^{1}$, Cierna $\mathrm{Z}^{2}$, Ferianec $\mathrm{V}^{1}$ \\ 2nd Department of Gynecology and Obstetrics, Faculty of Medicine, Comenius University, \\ Bratislava, Slovakia. ferianec@gmail.com
}

\begin{abstract}
OBJECTIVES: To analyze a rare triad of intracranial fetal pathologies and clinical study of the novel defined sequence pathogenesis based on prenatal and postmortem findings.

METHODS: Complex multidisciplinary clinical analysis and review of up-to-date literature.

RESULTS: In an 18-gestational-week fetus the screening ultrasound scan resembled the semilobar type of holoprosencephaly and oral tumor. After the indicated termination of pregnancy, the histopathology results confirmed another pathologies - oral meningoencephalocele, teratoma of the sellar area and large arachnoidal cyst of the anterior cerebral fossa. The surprising final results were evaluated by specialists in prenatal diagnosis, histopathology, genetics, neurology, and radiology.

CONCLUSION: We defined the final diagnosed triad oral meningoencephalocele - intracranial sellar teratomaarachnoidal cyst as a novel sequence defect malformation. In the detailed sequence pathogenesis, the intracranial sellar teratoma created an aperture for meningoencephalocele in the cranial base and the arachnoidal cyst facilitated, by its growth and pressure, the protrusion of the brain tissues (Fig. 4, Ref. 10). Text in PDF www.elis.sk. KEY WORDS: arachnoidal cyst, fetal, holoprosencephaly, meningoencephalocele, oral, sequence, teratoma.
\end{abstract}

\section{Introduction}

Congenital anomalies of the brain occur as independent defects, sequences or syndromes. Sequences are developmental disturbances caused by the cascade of events (1).

The rarest prenatally diagnosed neural tube defects are located in the sphenoidal area. Oral meningoencephalocele is usually diagnosed postnatally as a focal nasopharyngeal defect. The clinical presentation is variable, it may be asymptomatic or may include upper airways symptoms. Estimated incidence is 1:35,000 pregnancies. Prenatal diagnosis is extremely rare, and polyhydramnios is a common sign. In the differential diagnosis, epignathus and epulis should be ruled out. The prognosis depends on the extent of the brain involvement and associated anomalies. Postnatal treatment is surgical by the trans-sphenoidal approach. Sphenoidal encephalocele was also described within the Morning-Glory syndrome $(2,3)$.

Intracranial cystic formations may be classified into three different categories: extra-axial, intracerebral, and intraventricular. The most common prenatally diagnosed extra-axial cystic malformation is an arachnoidal cyst. Primary (congenital) arachnoi-

${ }^{1}$ 2nd Department of Gynecology and Obstetrics, Faculty of Medicine, Comenius University, Bratislava, Slovakia, and ${ }^{2}$ Institute of Pathological Anatomy, Faculty of Medicine, Comenius University, Bratislava, Slovakia

Address for correspondence: $\mathrm{V}$. Ferianec, MD, PhD, 2nd Department of Gynecology and Obstetrics, Faculty of Medicine, Comenius University, Ruzinovska 6, SK-826 06 Bratislava, Slovakia.

Phone: +421.905385670 dal cysts are usually filled with cerebrospinal fluid. The localization is between the dura and the brain substance, without a connection with the subarachnoidal space. Secondary (acquired) arachnoidal cysts result from hemorrhage or trauma. The cysts are predominantly on the surface of the brain and in the main brain fissures, anterior and middle cranial fossae. The prognosis depends on the location, volume and growth of the cyst during the brain development. Differential diagnosis includes holoprosencephaly, porencephaly, choroid plexus cysts, and venous aneurysms (4-8).

Although uncommon in the general population, intracranial teratoma is one of the most common neoplasms of the fetal brain. These intracranial germ cell tumors usually originate from all three embryonic layers and its typically mixed density is caused by extremely heterogeneous histological components $(9,10)$.

\section{Methods}

Complex multidisciplinary clinical analysis and review of up-to-date literature.

\section{Results}

In the 18-gestational-week fetus, our screening ultrasound examination revealed a large anechogenic cyst in the anterior and middle cranial fossae (Fig. 1) and a "sack-like" tumor measuring $26 \times 19 \mathrm{~mm}$ and mixed echogenicity protruded from the oral cavity (Fig. 2). The frontal brain structures were not visible, the posterior fossa, posterior cornua of the lateral ventricles as well as other fetal 


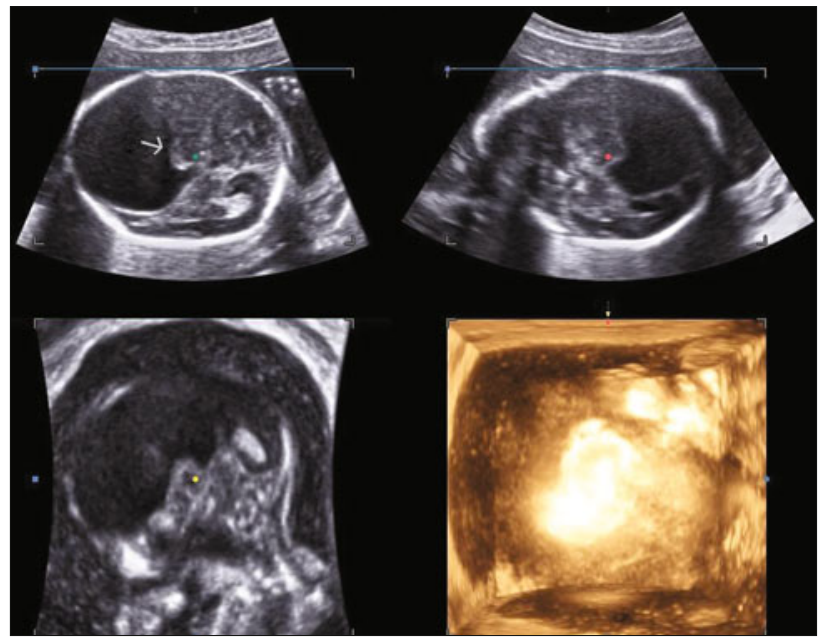

Fig. 1. Transabdominal 3D ultrasound, multiplanar view : large anechogenic cystic formation involving the whole anterior and middle cranium fossae ( arachnoidal cyst). Arrow - the teratoma of the sellar region defined primary as the rests of the thalami (pseudoholoprosencephaly) (3D Ultrasound analysis in transversal, frontal and midsagittal plane)

morphology were intact. An ultrasound image resembled the semilobar type of holoprosencephaly with the typically fused thalami protruded into the monoventricular cavity (Fig. 1 arrow). MRI scan confirmed the ultrasound findings. Because of the suggested poor prognosis, the pregnancy was terminated. The karyotype of the fetus was normal, and microarrays did not detect any pathology. The histopathology results were surprising and confirmed new pathologies clarifying the novel unique sequence pathogenesis. The sac in the oral cavity was defined as meningoencephalocele (Figs 3 and 4), all structures of the brain were present. Surprisingly, no signs of holoprosencephaly were confirmed. A histopathologist described the state as follows: mature teratoma of the sellar area and oral cavity, large arachnoidal cyst of the anterior cerebral fossa and oral meningoencephalocele (Fig. 4). The final results were evaluated and consulted with specialists in prenatal diagnosis, histopathology, genetics, neurology, and radiology.

\section{Discussion}

In up-to date scientific literature fetal oral trans-sphenoidal meningoencephalocele was never reported, three analyzed intracranial pathologies were never described together, nor such a sequence defect. The diagnosis of the three mentioned pathologies in the 18-week fetus (sellar teratoma, arachnoidal cyst, oral transsphenoidal meningoencephalocele) represents the type of novel sequence. As a pathogenesis, we described that the intracranial sellar teratoma created an aperture for meningoencephalocele in the cranial base and the arachnoidal cyst facilitated the protrusion by its growth and pressure. The amniotic fluid index was normal in this case. This is also a rare condition by the oral fetal masses. We assume, that the protrusion of the tumor in front of the mouth enabled normal fetal swallowing and circulation of the amniotic

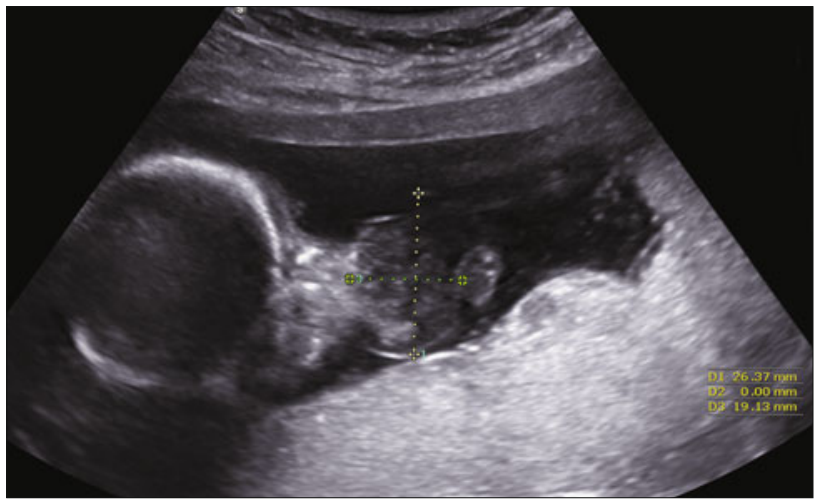

Fig. 2. Transabdominal ultrasound: A "sack" like oval formation of cca $26 \times 19 \mathrm{~mm}$ and mixed echogenity in front of the oral cavity (orally herniated meningoencephalocele)

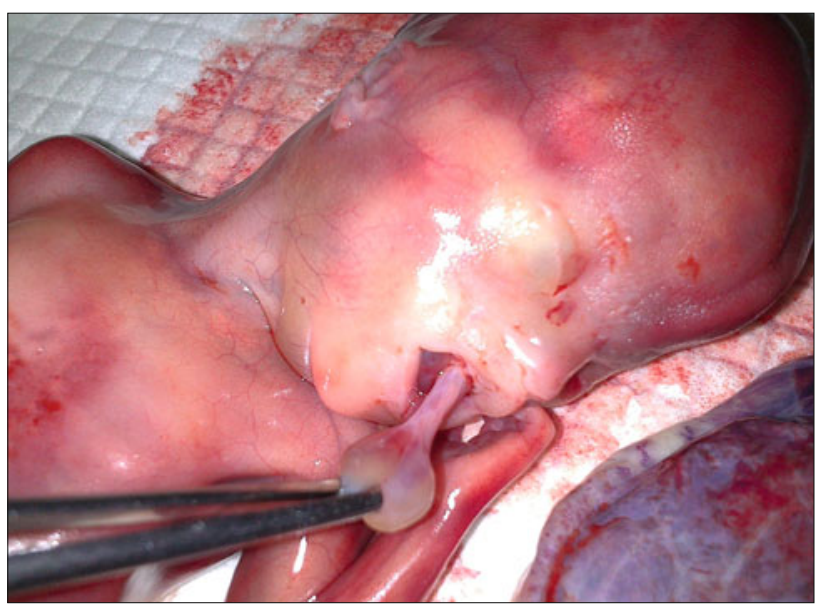

Fig. 3. Postmortem macroscopic view of the protruded fetal oral menigoencephalocele - correlation with prenatal ultrasound scan in Figure 2. The protrusion of the oral meningoencephalic sack was the result of the sequence pathogenesis - the sellar teratoma created an aperture in the cranial base and the arachnoidal cyst facilitated the protrusion of the brain tissues in to the oral cavity.

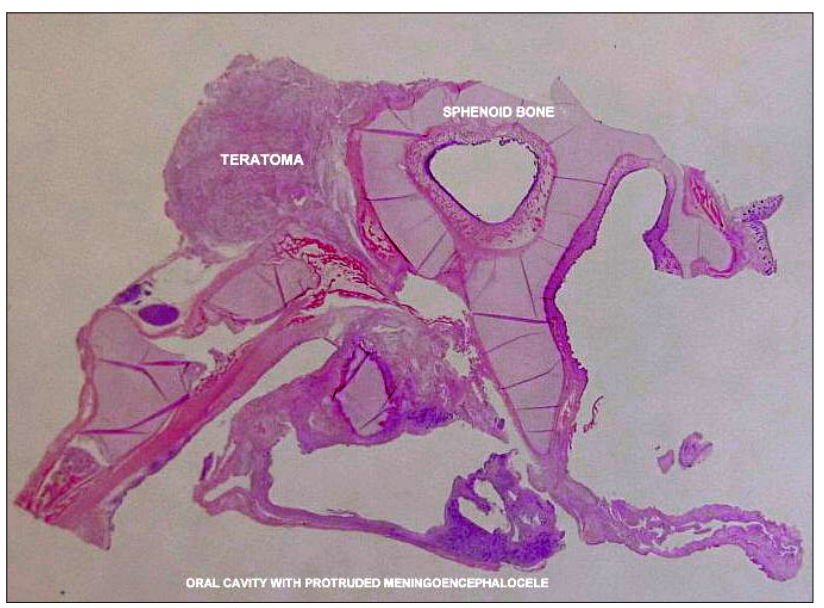

Fig. 4. Histopathological slice of the cranial base: The teratoma enabled the protrusion of the meningoencephalocele in front of the sphenoid bone to the oral cavity of the fetus. 


\section{2-274}

fluid. The histopathological slice of the cranial base with teratoma and meningoencephalocele is pictured in the Figure 4.

The cases of "pseudo" or "mimic" diagnoses in prenatal diagnosis are not rare. In our study the "pseudoholoprosencephaly" ultrasound picture mimicked the true pathologies. Even though the intracranial teratoma was not initially described on prenatal ultrasound or MRI, retrospectively we were able to recognize the teratoma-corresponding structure on the scans. We initially defined the structure as a remnant of the prosencephalon (Fig. 3 arrow). The final diagnosis was established due to the histopathological results. Thus, a precise critical verification of the definite diagnosis should be the gold standard. We would like to emphasize the importance of the mutual correlation between the prenatal and histopathological diagnosis.

\section{Learning points}

Sequences are developmental disturbances caused by the cascade of events.

The rarest prenatally diagnosed neural tube defects are located in the sphenoidal area.

Oral meningoencephalocele was never diagnosed prenatally.

Intracranial sellar teratoma of the fetus can create in the sequence an aperture for meningoencephalocele in the cranial base and the presence of an arachnoidal cyst facilitates its oral protrusion by the pressure and growth.

The cases of "pseudo" or "mimic" diagnoses in prenatal diagnosis are not rare. Arachnoidal cyst with cranial base tumor can mimic the picture of semilobar holoprosencephaly in prenatal ultrasound scan ("pseudoholoprosencephaly")

A precise critical verification of the definite diagnosis and correlation between the prenatal and histopathological diagnosis should be the gold standard.

\section{References}

1. Parthasarathi A, Kishor VH. Trans-sellar transsphenoidal nasopharyngeal encephalocele. J Evol Med Dent Sci 2013; 12: 10092-10097.

2. Itakura T, Miyamoto K, Uematsu $\mathbf{Y}$ et al. Bilateral morning glory syndrome associated with sphenoid encephalocele. J Neurosurg 1992; 77: 949-951.

3. Carlan SJ, Angel JL, Leo J et al. Cephalocele involving the oral cavity. Obstet Gynecol 1990; 75: 494-496.

4. Raam MS, Solomon BD, Muenke M. Holoprosencephaly: a guide to diagnosis and clinical management. Indian Pediatrics 2011; 48: 457-466.

5. Joó Gábor J, Beke A, Papp C et al. Prenatal diagnosis, phenotypic and obstetric characteristics of holoprosencephaly. Fetal Diagn Ther 2005; 20: $161-166$

6. Bretelle F, Senat M-V, Bernard J-P et al. First-trimester diagnosis of fetal arachnoid cyst: prenatal implication. Ultrasound Obstet Gynecol 2002; 20: 400-402.

7. Gedikbasi A, Palabiyik F, Oztarhan A et al. Prenatal diagnosis of a suprasellar arachnoid cyst with 2- and 3-dimensional sonography and fetal magnetic resonance imaging. Difficulties in management and review of the literature. J Ultrasound Med 2010; 29: 1487-1493.

8. Monteiro M, de Albuquerque AC, Nobre MC et al. Transsphenoidal transpalatal meningoencephalocele. Arq Neuropsiquiatr 2006; 64: 624-627.

9. Cassart M, Bosson N, Garel C et al. Fetal intracranial tumors: a review of 27 cases. Eur Radiol 2008; 18: 2060-2066.

10. Arslan E, Usul H, Baykal S et al. Massive congenital intracranial immature teratoma of the lateral ventricle with retroorbital extension. Pediatr Neurosurg 2007; 43: 338-342.

Received February 4, 2018. Accepted February 27, 2018. 